\title{
@creative
}

\section{Mixed fault diameter of Cartesian graph bundles II}

\author{
Rija Erveš * \\ FCE, University of Maribor, \\ Smetanova 17, Maribor 2000, Slovenia \\ Janez Žerovnik \\ FME, University of Ljubljana, \\ Aškerčeva 6, Ljubljana 1000, Slovenia
}

Received 2 October 2012, accepted 13 December 2012, published online 23 January 2015

\begin{abstract}
The mixed fault diameter $\mathcal{D}_{(p, q)}(G)$ is the maximum diameter among all subgraphs obtained from graph $G$ by deleting $p$ vertices and $q$ edges. A graph is $(p, q)+$ connected if it remains connected after removal of any $p$ vertices and any $q$ edges. Let $F$ be a connected graph with the diameter $\mathcal{D}(F)>1$, and $B$ be $(p, q)+$ connected graph. Upper bounds for the mixed fault diameter of Cartesian graph bundle $G$ with fibre $F$ over the base graph $B$ are given. We prove that if $q>0$, then $\mathcal{D}_{(p+1, q)}(G) \leq \mathcal{D}(F)+\mathcal{D}_{(p, q)}(B)$, and if $q=0$ and $p>0$, then $\mathcal{D}_{(p+1,0)}(G) \leq \mathcal{D}(F)+\max \left\{\mathcal{D}_{(p, 0)}(B), \mathcal{D}_{(p-1,1)}(B)\right\}$.
\end{abstract}

Keywords: Mixed fault diameter, Cartesian graph bundle, interconnection network, fault tolerance.

Math. Subj. Class.: 05C12, 05C40, 68M10, 68M15, 68R10

\section{Introduction}

Graph products and bundles belong to a class of frequently studied interconnection network topologies. For example meshes, tori, hypercubes and some of their generalizations are Cartesian products. It is less known that some other well-known interconnection network topologies are Cartesian graph bundles, for example twisted hypercubes [9, 12] and multiplicative circulant graphs [23].

\footnotetext{
${ }^{*}$ Both authors are also researchers at Institute of mathematics, physics and mechanics. The research supported in part by the Research agency of Slovenia ARRS.

E-mail addresses: rija.erves@um.si (Rija Erveš), janez.zerovnik@fs.uni-lj.si, janez.zerovnik@imfm.si (Janez Žerovnik)
} 
In the design of large interconnection networks several factors have to be taken into account. A usual constraint is that each processor can be connected to a limited number of other processors and that the delays in communication must not be too long. Furthermore, an interconnection network should be fault tolerant, because practical communication networks are exposed to failures of network components. Both failures of nodes and failures of connections between them happen and it is desirable that a network is robust in the sense that a limited number of failures does not break down the whole system. A lot of work has been done on various aspects of network fault tolerance, see for example the survey [8] and the more recent papers [16, 24, 26]. In particular the fault diameter with faulty vertices, which was first studied in [18], and the edge fault diameter have been determined for many important networks recently [2, 3, 4, 5, 10, 11, 19, 25]. Usually either only edge faults or only vertex faults are considered, while the case when both edges and vertices may be faulty is studied rarely. For example, [16, 24] consider Hamiltonian properties assuming a combination of vertex and edge faults. In recent work on fault diameter of Cartesian graph products and bundles [2, 3, 4, 5], analogous results were found for both fault diameter and edge fault diameter. However, the proofs for vertex and edge faults are independent, and our effort to see how results in one case may imply the others was not successful. A natural question is whether it is possible to design a uniform theory that covers simultaneous faults of vertices and edges. Some basic results on edge, vertex and mixed fault diameters for general graphs appear in [6]. In order to study the fault diameters of graph products and bundles under mixed faults, it is important to understand generalized connectivities. Mixed connectivity which generalizes both vertex and edge connectivity, and some basic observations for any connected graph are given in [13]. We are not aware of any earlier work on mixed connectivity. A closely related notion is the connectivity pairs of a graph [7], but after Mader [20] showed the claimed proof of generalized Menger's theorem is not valid, work on connectivity pairs seems to be very rare.

An upper bound for the mixed fault diameter of Cartesian graph bundles is given in [14] that in some case also improves previously known results on vertex and edge fault diameters on these classes of Cartesian graph bundles [2, 5]. However these results address only the number of faults given by the connectivity of the fibre (plus one vertex), while the connectivity of the graph bundle can be much higher when the connectivity of $B$ is substantial. It seems obvious that the upper bound from [14] can be improved. In this paper we provide an upper bound that takes into account the mixed connectivity of the base graph $B$, i.e. the number of faults allowed is given by the connectivity of the base graph (plus one vertex), thus complementing the result of [14]. We show by examples that the bounds of the new result are tight. In addition, in some cases Theorem 4.6 also improves previously known results on vertex and edge fault diameters on these classes of Cartesian graph bundles $[2,5]$.

The rest of the paper is organized as follows. General definitions, in particular of the connectivities, are given in section Preliminaries. The third section introduces graph bundles and recalls relevant previous results. In Section 4, the proof of the main theorem is given, followed by a short discussion.

\section{Preliminaries}

A simple graph $G=(V, E)$ is determined by a vertex set $V=V(G)$ and a set $E=E(G)$ of (unordered) pairs of vertices, called edges. As usual, we will use the short notation 
$u v$ for edge $\{u, v\}$. For an edge $e=u v$ we call $u$ and $v$ its endpoints. It is sometimes convenient to consider the union of elements of a graph, $S(G)=V(G) \cup E(G)$. Given $X \subseteq S(G)$ then $S(G) \backslash X$ is a subset of elements of $G$. However, note that in general $S(G) \backslash X$ may not induce a graph. As we need notation for subgraphs with some missing (faulty) elements, we formally define $G \backslash X$, the subgraph of $G$ after deletion of $X$, as follows:

Definition 2.1. Let $X \subseteq S(G)$, and $X=X_{E} \cup X_{V}$, where $X_{E} \subseteq E(G)$ and $X_{V} \subseteq V(G)$. Then $G \backslash X$ is the subgraph of $\left(V(G), E(G) \backslash X_{E}\right)$ induced on vertex set $V(G) \backslash X_{V}$.

A walk between vertices $x$ and $y$ is a sequence of vertices and edges $v_{0}, e_{1}, v_{1}, e_{2}, v_{2}$, $\ldots, v_{k-1}, e_{k}, v_{k}$ where $x=v_{0}, y=v_{k}$, and $e_{i}=v_{i-1} v_{i}$ for each $i$. A walk with all vertices distinct is called a path, and the vertices $v_{0}$ and $v_{k}$ are called the endpoints of the path. The length of a path $P$, denoted by $\ell(P)$, is the number of edges in $P$. The distance between vertices $x$ and $y$, denoted by $d_{G}(x, y)$, is the length of a shortest path between $x$ and $y$ in $G$. If there is no path between $x$ and $y$ we write $d_{G}(x, y)=\infty$. The diameter of a connected graph $G, \mathcal{D}(G)$, is the maximum distance between any two vertices in $G$. A path $P$ in $G$, defined by a sequence $x=v_{0}, e_{1}, v_{1}, e_{2}, v_{2}, \ldots, v_{k-1}, e_{k}, v_{k}=y$ can alternatively be seen as a subgraph of $G$ with $V(P)=\left\{v_{0}, v_{1}, v_{2}, \ldots, v_{k}\right\}$ and $E(P)=$ $\left\{e_{1}, e_{2}, \ldots, e_{k}\right\}$. Note that the reverse sequence gives rise to the same subgraph. Hence we use $P$ for a path either from $x$ to $y$ or from $y$ to $x$. A graph is connected if there is a path between each pair of vertices, and is disconnected otherwise. In particular, $K_{1}$ is by definition disconnected. The connectivity (or vertex connectivity) $\kappa(G)$ of a connected graph $G$, other than a complete graph, is the smallest number of vertices whose removal disconnects $G$. For complete graphs is $\kappa\left(K_{n}\right)=n-1$. We say that $G$ is $k$-connected (or $k$-vertex connected) for any $0<k \leq \kappa(G)$. The edge connectivity $\lambda(G)$ of a connected graph $G$, is the smallest number of edges whose removal disconnects $G$. A graph $G$ is said to be $k$-edge connected for any $0<k \leq \lambda(G)$. It is well known that (see, for example, [1], page 224) $\kappa(G) \leq \lambda(G) \leq \delta_{G}$, where $\delta_{G}$ is smallest vertex degree of $G$. Thus if a graph $G$ is $k$-connected, then it is also $k$-edge connected. The reverse does not hold in general.

Here we are interested in mixed connectivity that generalizes both vertex and edge connectivity. Note that the definition used here slightly differs from the definition used in a previous work [13].

Definition 2.2. Let $G$ be any connected graph. A graph $G$ is $(p, q)+$ connected, if $G$ remains connected after removal of any $p$ vertices and any $q$ edges.

We wish to remark that the mixed connectivity studied here is closely related to connectivity pairs as defined in [7]. Briefly speaking, a connectivity pair of a graph is an ordered pair $(k, \ell)$ of two integers such that there is some set of $k$ vertices and $\ell$ edges whose removal disconnects the graph and there is no set of $k-1$ vertices and $\ell$ edges or of $k$ vertices and $\ell-1$ edges with this property. Clearly $(k, \ell)$ is a connectivity pair of $G$ exactly when: (1) $G$ is $(k-1, \ell)+$ connected, (2) $G$ is $(k, \ell-1)+$ connected, and (3) $G$ is not $(k, \ell)+$ connected. In fact, as shown in [13], (2) implies (1), so $(k, \ell)$ is a connectivity pair exactly when (2) and (3) hold.

From the definition we easily observe that any connected graph $G$ is $(0,0)+$ connected, $(p, 0)+$ connected for any $p<\kappa(G)$ and $(0, q)+$ connected for any $q<\lambda(G)$. In our notation $(i, 0)+$ connected is the same as $(i+1)$-connected, i.e. the graph remains connected after 
removal of any $i$ vertices. Similarly, $(0, j)+$ connected means $(j+1)$-edge connected, i.e. the graph remains connected after removal of any $j$ edges.

Clearly, if $G$ is a $(p, q)+$ connected graph, then $G$ is $\left(p^{\prime}, q^{\prime}\right)+$ connected for any $p^{\prime} \leq p$ and any $q^{\prime} \leq q$. Furthermore, for any connected graph $G$ with $k<\kappa(G)$ faulty vertices, at least $k$ edges are not working. Roughly speaking, graph $G$ remains connected if any faulty vertex in $G$ is replaced with a faulty edge. It is known [13] that if a graph $G$ is $(p, q)+$ connected and $p>0$, then $G$ is $(p-1, q+1)+$ connected. Hence for $p>0$ we have a chain of implications: $(p, q)+$ connected $\Longrightarrow(p-1, q+1)+$ connected $\Longrightarrow \ldots \Longrightarrow$ $(1, p+q-1)+$ connected $\Longrightarrow(0, p+q)+$ connected, which generalizes the well-known proposition that any $k$-connected graph is also $k$-edge connected. Therefore, a graph $G$ is $(p, q)+$ connected if and only if $p<\kappa(G)$ and $p+q<\lambda(G)$.

Note that by our definition the complete graph $K_{n}, n \geq 2$, is $(n-2,0)+$ connected, and hence $(i, j)+$ connected for any $i+j \leq n-2$. Graph $K_{2}$ is $(0,0)+$ connected, and mixed connectivity of $K_{1}$ is not defined.

If for a graph $G \kappa(G)=\lambda(G)=k$, then $G$ is $(i, j)+$ connected exactly when $i+j<k$. However, if $2 \leq \kappa(G)<\lambda(G)$, the question whether $G$ is $(i, j)+$ connected for $1 \leq$ $i<\kappa(G) \leq i+j<\lambda(G)$ is not trivial. The example below shows that in general the knowledge of $\kappa(G)$ and $\lambda(G)$ is not enough to decide whether $G$ is $(i, j)+$ connected.

Example 2.3. For graphs on Fig. 1 we have $\kappa\left(G_{1}\right)=\kappa\left(G_{2}\right)=2$ and $\lambda\left(G_{1}\right)=\lambda\left(G_{2}\right)=$ 3 . Both graphs are $(1,0)+$ connected $\Longrightarrow(0,1)+$ connected, and $(0,2)+$ connected. Graph $G_{1}$ is not $(1,1)+$ connected, while graph $G_{2}$ is.
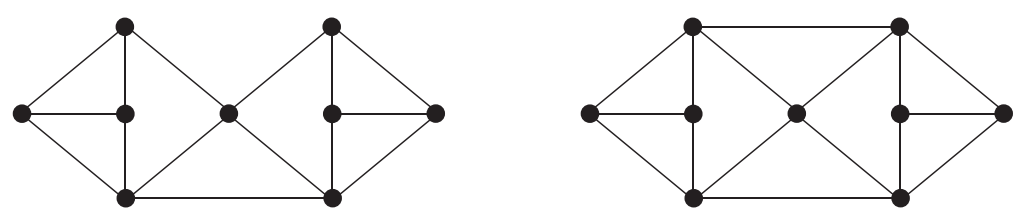

Figure 1: Graphs $G_{1}$ and $G_{2}$ from Example 2.3.

Definition 2.4. Let $G$ be a $k$-edge connected graph and $0 \leq a<k$. The a-edge fault diameter of $G$ is

$$
\mathcal{D}_{a}^{E}(G)=\max \{\mathcal{D}(G \backslash X)|X \subseteq E(G),| X \mid=a\} .
$$

Definition 2.5. Let $G$ be a $k$-connected graph and $0 \leq a<k$. The a-fault diameter (or a-vertex fault diameter) of $G$ is

$$
\mathcal{D}_{a}^{V}(G)=\max \{\mathcal{D}(G \backslash X)|X \subseteq V(G),| X \mid=a\} .
$$

Note that $\mathcal{D}_{a}^{E}(G)$ is the largest diameter among the diameters of subgraphs of $G$ with $a$ edges deleted, and $\mathcal{D}_{a}^{V}(G)$ is the largest diameter over all subgraphs of $G$ with $a$ vertices deleted. In particular, $\mathcal{D}_{0}^{E}(G)=\mathcal{D}_{0}^{V}(G)=\mathcal{D}(G)$, the diameter of $G$. For $p \geq \kappa(G)$ and for $q \geq \lambda(G)$ we set $\mathcal{D}_{p}^{V}(G)=\infty, \mathcal{D}_{q}^{E}(G)=\infty$, as some of the subgraphs are not vertex connected or edge connected, respectively.

It is known [6] that for any connected graph $G$ the inequalities below hold. 

1. $\mathcal{D}(G)=\mathcal{D}_{0}^{E}(G) \leq \mathcal{D}_{1}^{E}(G) \leq \mathcal{D}_{2}^{E}(G) \leq \ldots \leq \mathcal{D}_{\lambda(G)-1}^{E}(G)<\infty$.
2. $\mathcal{D}(G)=\mathcal{D}_{0}^{V}(G) \leq \mathcal{D}_{1}^{V}(G) \leq \mathcal{D}_{2}^{V}(G) \leq \ldots \leq \mathcal{D}_{\kappa(G)-1}^{V}(G)<\infty$.

Definition 2.6. Let $G$ be a $(p, q)+$ connected graph. The $(p, q)$-mixed fault diameter of $G$ is

$$
\mathcal{D}_{(p, q)}(G)=\max \{\mathcal{D}(G \backslash(X \cup Y))|X \subseteq V(G), Y \subseteq E(G),| X|=p,| Y \mid=q\}
$$

Note that by Definition 2.6 the endpoints of edges of set $Y$ can be in $X$. In this case we may get the same subgraph of $G$ by deleting $p$ vertices and fewer than $q$ edges. It is however not difficult to see that the diameter of such subgraph is smaller than or equal to the diameter of some subgraph of $G$ where exactly $p$ vertices and exactly $q$ edges are deleted. So the condition that the endpoints of edges of set $Y$ are not in $X$ need not to be included in Definition 2.6. The mixed fault diameter $\mathcal{D}_{(p, q)}(G)$ is the largest diameter among the diameters of all subgraphs obtained from $G$ by deleting $p$ vertices and $q$ edges, hence $\mathcal{D}_{(0,0)}(G)=\mathcal{D}(G), \mathcal{D}_{(0, a)}(G)=\mathcal{D}_{a}^{E}(G)$ and $\mathcal{D}_{(a, 0)}(G)=\mathcal{D}_{a}^{V}(G)$.

Let $\mathcal{H}_{a}^{V}=\{G \backslash X|X \subseteq V(G)| X \mid,=a\}$ and $\mathcal{H}_{b}^{E}=\{G \backslash X|X \subseteq E(G)| X \mid,=b\}$. It is easy to see that

1. $\max \left\{\mathcal{D}_{b}^{E}(H) \mid H \in \mathcal{H}_{a}^{V}\right\}=\mathcal{D}_{(a, b)}(G)$,

2. $\max \left\{\mathcal{D}_{a}^{V}(H) \mid H \in \mathcal{H}_{b}^{E}\right\}=\mathcal{D}_{(a, b)}(G)$.

In previous work [6] on vertex, edge and mixed fault diameters of connected graphs the following theorem has been proved.

Theorem 2.7. Let $G$ be $(p, q)+$ connected graph and $p>0$.

- If $q>0$, then $\mathcal{D}_{p+q}^{E}(G) \leq \mathcal{D}_{(1, p+q-1)}(G) \leq \cdots \leq \mathcal{D}_{(p, q)}(G)$.

- If $q=0$, then $\mathcal{D}_{p}^{E}(G) \leq \mathcal{D}_{(1, p-1)}(G) \leq \cdots \leq \mathcal{D}_{(p-1,1)}(G) \leq \mathcal{D}_{p}^{V}(G)+1$.

\section{Mixed fault diameter of Cartesian graph bundles}

Cartesian graph bundles are a generalization of Cartesian graph products, first studied in [21, 22]. Let $G_{1}$ and $G_{2}$ be graphs. The Cartesian product of graphs $G_{1}$ and $G_{2}, G=$ $G_{1} \square G_{2}$, is defined on the vertex set $V\left(G_{1}\right) \times V\left(G_{2}\right)$. Vertices $\left(u_{1}, v_{1}\right)$ and $\left(u_{2}, v_{2}\right)$ are adjacent if either $u_{1} u_{2} \in E\left(G_{1}\right)$ and $v_{1}=v_{2}$ or $v_{1} v_{2} \in E\left(G_{2}\right)$ and $u_{1}=u_{2}$. For further reading on graph products we recommend [15].

Definition 3.1. Let $B$ and $F$ be graphs. A graph $G$ is a Cartesian graph bundle with fibre $F$ over the base graph $B$ if there is a graph map $p: G \rightarrow B$ such that for each vertex $v \in V(B), p^{-1}(\{v\})$ is isomorphic to $F$, and for each edge $e=u v \in E(B), p^{-1}(\{e\})$ is isomorphic to $F \square K_{2}$.

More precisely, the mapping $p: G \rightarrow B$ maps graph elements of $G$ to graph elements of $B$, i.e. $p: V(G) \cup E(G) \rightarrow V(B) \cup E(B)$. In particular, here we also assume that the vertices of $G$ are mapped to vertices of $B$ and the edges of $G$ are mapped either to vertices or to edges of $B$. We say an edge $e \in E(G)$ is degenerate if $p(e)$ is a vertex. Otherwise we call it nondegenerate. The mapping $p$ will also be called the projection (of the bundle $G$ to its base $B$ ). Note that each edge $e=u v \in E(B)$ naturally induces an 
isomorphism $\varphi_{e}: p^{-1}(\{u\}) \rightarrow p^{-1}(\{v\})$ between two fibres. It may be interesting to note that while it is well-known that a graph can have only one representation as a product (up to isomorphism and up to the order of factors) [15], there may be many different graph bundle representations of the same graph [29]. Here we assume that the bundle representation is given. Note that in some cases finding a representation of $G$ as a graph bundle can be found in polynomial time $[17,27,28,29,30,31]$. For example, one of the easy classes are the Cartesian graph bundles over triangle-free base [17]. Note that a graph bundle over a tree $T$ (as a base graph) with fibre $F$ is isomorphic to the Cartesian product $T \square F$ (not difficult to see, appears already in [21]), i.e. we can assume that all isomorphisms $\varphi_{e}$ are identities. For a later reference note that for any path $P \subseteq B, p^{-1}(P)$ is a Cartesian graph bundle over the path $P$, and one can define coordinates in the product $P \square F$ in a natural way.

In recent work on fault diameter of Cartesian graph products and bundles [2, 3, 4, 5], analogous results were found for both fault diameter and edge fault diameter.

Theorem 3.2. [2] Let $G$ be a Cartesian bundle with fibre F over the base graph B, graph $F$ be $(a, 0)+$ connected and graph $B$ be $(b, 0)+$ connected. Then

$$
\mathcal{D}_{a+b+1}^{V}(G) \leq \mathcal{D}_{a}^{V}(F)+\mathcal{D}_{b}^{V}(B)+1
$$

Theorem 3.3. [5] Let $G$ be a Cartesian bundle with fibre $F$ over the base graph B, graph $F$ be $(0, a)+$ connected and graph $B$ be $(0, b)+$ connected. Then

$$
\mathcal{D}_{a+b+1}^{E}(G) \leq \mathcal{D}_{a}^{E}(F)+\mathcal{D}_{b}^{E}(B)+1 .
$$

Before writing a theorem on bounds for the mixed fault diameter we recall a theorem on mixed connectivity.

Theorem 3.4. [13] Let $G$ be a Cartesian graph bundle with fibre $F$ over the base graph $B$, graph $F$ be $\left(p_{F}, q_{F}\right)+$ connected and graph $B$ be $\left(p_{B}, q_{B}\right)+$ connected. Then Cartesian graph bundle $G$ is $\left(p_{F}+p_{B}+1, q_{F}+q_{B}\right)+$ connected.

In recent work [14], an upper bound for the mixed fault diameter of Cartesian graph bundles, $\mathcal{D}_{(p+1, q)}(G)$, in terms of mixed fault diameter of the fibre and diameter of the base graph is given. Theorem 3.5 improves results 3.2 and 3.3 for $b=0$.

Theorem 3.5. [14] Let $G$ be a Cartesian graph bundle with fibre $F$ over the base graph $B$, where graph $F$ is $(p, q)+$ connected, $p+q>0$, and $B$ is a connected graph with diameter $\mathcal{D}(B)>1$. Then we have:

- If $q>0$, then $\mathcal{D}_{(p+1, q)}(G) \leq \mathcal{D}_{(p, q)}(F)+\mathcal{D}(B)$.

- If $q=0$, then $\mathcal{D}_{p+1}^{V}(G) \leq \max \left\{\mathcal{D}_{p}^{V}(F), \mathcal{D}_{(p-1,1)}(F)\right\}+\mathcal{D}(B)$.

Here we prove a similar result for an upper bound for the mixed fault diameter of Cartesian graph bundles, $\mathcal{D}_{(p+1, q)}(G)$, in terms of diameter of the fibre and mixed fault diameter of the base graph. We consider mixed fault diameter of Cartesian graph bundle $G$ with connected fibre $F$. If the graph $B$ is $(p, q)+$ connected then Cartesian graph bundle with connected fibre $F$ over the base graph $B$ is at least $(p+1, q)+$ connected. Theorem 4.6 improves results 3.2 and 3.3 for $a=0$. 


\section{Proof of the main theorem}

Before stating and proving the main theorem, we prove several lemmas and introduce some notation used in this section.

Let $G$ be a Cartesian graph bundle with fibre $F$ over the base graph $B$. The fibre of vertex $x \in V(G)$ is denoted by $F_{x}$, formally, $F_{x}=p^{-1}(\{p(x)\})$. We will also use notation $F(u)$ for the fibre of the vertex $u \in V(B)$, i.e. $F(u)=p^{-1}(\{u\})$. Note that $F_{x}=F(p(x))$. We will also use shorter notation $x \in F(u)$ for $x \in V(F(u))$.

Let $u, v \in V(B)$ be distinct vertices, and $Q$ be a path from $u$ to $v$ in $B$, and $x \in F(u)$. Then the lift of the path $Q$ to the vertex $x \in V(G), \tilde{Q}_{x}$, is the path from $x \in F(u)$ to a vertex in $F(v)$, such that $p\left(\tilde{Q}_{x}\right)=Q$ and $\ell\left(\tilde{Q}_{x}\right)=\ell(Q)$. Let $x, x^{\prime} \in F(u)$. Then $\tilde{Q}_{x}$ and $\tilde{Q}_{x^{\prime}}$ have different endpoints in $F(v)$ and are disjoint paths if and only if $x \neq x^{\prime}$. In fact, two lifts $\tilde{Q}_{x}$ and $\tilde{Q}_{x^{\prime}}$ are either disjoint $\tilde{Q}_{x} \cap \tilde{Q}_{x^{\prime}}=\emptyset$ or equal, $\tilde{Q}_{x}=\tilde{Q}_{x^{\prime}}$. We will also use notation $\tilde{Q}$ for lifts of the path $Q$ to any vertex in $F(u)$.

Let $Q$ be a path from $u$ to $v$ and $e=u w \in E(Q)$. We will use notation $Q \backslash e$ for the subpath from $w$ to $v$, i.e. $Q \backslash e=Q \backslash\{u, e\}=Q \backslash\{u\}$.

Let $G$ be a graph and $X \subseteq S(G)$ be a set of elements of $G$. A path $P$ from a vertex $x$ to a vertex $y$ avoids $X$ in $G$, if $S(P) \cap X=\emptyset$, and it internally avoids $X$, if $(S(P) \backslash\{x, y\}) \cap X=\emptyset$.

We will use Lemma 4.1 in following proofs.

Lemma 4.1. Let $F$ and $B$ be connected graphs, $\mathcal{D}(F)>1$, and let $G$ be a Cartesian graph bundle with fibre $F$ over the base graph $B$. Let $x, y \in V(G)$ be two vertices, such that $p(x) \neq p(y)$, and let $Q$ be a path from $p(x)$ to $p(y)$ in $B$. Then there are (at least) two internally vertex-disjoint paths from $x$ to $y$ in $p^{-1}(Q)=F \square Q \subseteq G$ of lengths at most $\mathcal{D}(F)+\ell(Q)$.

Proof. Let $G$ be a Cartesian graph bundle with connected fibre $F, \mathcal{D}(F)>1$, over the connected base graph $B$. Let $x, y \in V(G), p(x) \neq p(y)$, and $Q$ be a path from $p(x)$ to $p(y)$ in $B$. Let $x^{\prime} \in F_{y}$ be the endpoint of $\tilde{Q}_{x}$.

- If $x^{\prime}=y$, then there are two paths

$$
P_{1}: x \stackrel{\tilde{Q}}{\rightarrow} y, P_{2}: x \rightarrow s \stackrel{\tilde{Q}}{\rightarrow} s^{\prime} \rightarrow y
$$

where $s \in F_{x}$ and $s^{\prime} \in F_{y}$ are neighbors of $x$ and $y$, respectively. Paths $P_{1}, P_{2}$ are internally vertex-disjoint paths from $x$ to $y$ in $p^{-1}(Q)$ and $\ell\left(P_{1}\right)=\ell(Q), \ell\left(P_{2}\right)=$ $1+\ell(Q)+1 \leq \mathcal{D}(F)+\ell(Q)$.

- If $x^{\prime} \neq y$, then there are two paths

$$
P_{1}: x \stackrel{\tilde{Q}}{\rightarrow} x^{\prime} \stackrel{P}{\rightarrow} y, P_{2}: x \stackrel{P^{\prime}}{\rightarrow} y^{\prime} \stackrel{\tilde{Q}}{\rightarrow} y
$$

where $P$ is a path from $x^{\prime}$ to $y$ inside of the fibre $F_{y}$ of length $\ell(P) \leq \mathcal{D}(F)$, $y^{\prime} \in F_{x}$ is the endpoint of $\tilde{Q}_{y}$ and $P^{\prime}$ is a path inside of the fibre $F_{x}$ of length $\ell\left(P^{\prime}\right) \leq \mathcal{D}(F)$. Paths $P_{1}, P_{2}$ are internally vertex-disjoint paths from $x$ to $y$ in $p^{-1}(Q)$ and $\ell\left(P_{i}\right) \leq \ell(Q)+\mathcal{D}(F), i=1,2$. 
Lemma 4.2. Let $G$ be a Cartesian graph bundle with fibre $F$ over the base graph $B$, the graph $F$ be a connected graph with diameter $\mathcal{D}(F)>1$, and the graph $B$ be $(p, 0)+$ connected, $p>0$. Then

$$
\mathcal{D}_{(p+1,0)}(G)=\mathcal{D}_{p+1}^{V}(G) \leq \mathcal{D}(F)+\max \left\{\mathcal{D}_{p}^{V}(B), \mathcal{D}_{(p-1,1)}(B)\right\} .
$$

Proof. Let $F$ be a connected graph, $\mathcal{D}(F)>1$, the graph $B$ be $(p, 0)+$ connected, $p>0$, and let $G$ be a Cartesian graph bundle with fibre $F$ over the base graph $B$. By Theorem 3.4, the Cartesian graph bundle $G$ is $(p+1,0)+$ connected. Let $X \subseteq V(G)$ be a set of faulty vertices, $|X|=p+1$, and let $x, y \in V(G) \backslash X$ be two distinct nonfaulty vertices in $G$. We shall consider the distance $d_{G \backslash X}(x, y)$. Note that as graph $B$ is $(p, 0)+$ connected and $p>0$, it is also $(p-1,1)+$ connected and $\mathcal{D}_{(p-1,1)}(B) \geq 2$.

- Suppose first that $x$ and $y$ are in the same fibre, i.e. $p(x)=p(y)$.

If $\left|X \cap V\left(F_{x}\right)\right|=0$, then $d_{G \backslash X}(x, y) \leq \mathcal{D}(F)$. If $\left|X \cap V\left(F_{x}\right)\right|>0$, then outside of fibre $F_{x}$ there are at most $p$ faulty vertices. As a graph $B$ is $(p, 0)+$ connected, there are at least $p+1$ neighbors of vertex $p(x)$ in $B$. Therefore there exist a neighbor $v$ of vertex $p(x)$ in $B$, such that $|X \cap F(v)|=0$, and there is a path $x \rightarrow x^{\prime} \stackrel{P}{\rightarrow} y^{\prime} \rightarrow y$, which avoids $X$, where $x^{\prime}, y^{\prime} \in F(v)$ and $\ell(P) \leq \mathcal{D}(F)$. Thus $d_{G \backslash X}(x, y) \leq$ $1+\mathcal{D}(F)+1 \leq \mathcal{D}(F)+\mathcal{D}_{(p-1,1)}(B)$.

- Now assume that $x$ and $y$ are in distinct fibres, i.e. $p(x) \neq p(y)$.

Denote $X_{B}=\{v \in V(B) \backslash\{p(x), p(y)\} ;|X \cap F(v)|>0\}$. We distinguish two cases.

1. If $p \leq\left|X_{B}\right| \leq p+1$, then let $X_{B}^{\prime} \subseteq X_{B}$ be an arbitrary subset of $X_{B}$ with $\left|X_{B}^{\prime}\right|=p$. The subgraph $B \backslash X_{B}^{\prime}$ is a connected graph and there exist a path $Q$ from $p(x)$ to $p(y)$ with $\ell(Q) \leq \mathcal{D}_{p}^{V}(B)$. In $p^{-1}(Q)$ there is at most one faulty vertex. By Lemma 4.1 there are two internally vertex-disjoint paths from $x$ to $y$ in $p^{-1}(Q)$ and at least one of them avoids the faulty element, thus $d_{G \backslash X}(x, y) \leq \ell(Q)+D(F) \leq \mathcal{D}_{p}^{V}(B)+D(F)$.

2. If $\left|X_{B}\right|<p$, then the subgraph $B \backslash X_{B}$ is (at least) (1,0)+connected, thus also $(0,1)+$ connected.

If the vertex $p(y)$ is not a neighbor of $p(x)$, then there is a path $Q$ from $p(x)$ to $p(y)$ in $B$ with $2 \leq \ell(Q) \leq \mathcal{D}_{p-1}^{V}(B) \leq \mathcal{D}_{p}^{V}(B)$ that internally avoids $X_{B}$. Let $v \in V(Q)$ be a neighbor of $p(x), e^{\prime}=p(x) v$. Then there is a path $x \rightarrow x^{\prime} \stackrel{P}{\rightarrow} y^{\prime} \stackrel{\tilde{Q} \backslash e^{\prime}}{\rightarrow} y$, which avoids $X$, where $x^{\prime}, y^{\prime} \in F(v)$ and $\ell(P) \leq \mathcal{D}(F)$. Thus $d_{G \backslash X}(x, y) \leq 1+\mathcal{D}(F)+\mathcal{D}_{p}^{V}(B)-1=\mathcal{D}(F)+\mathcal{D}_{p}^{V}(B)$.

If $e=p(x) p(y) \in E(B)$, then $B \backslash\left(X_{B} \cup\{e\}\right)$ is a connected graph and there is a path $Q^{\prime}$ from $p(x)$ to $p(y)$ with $2 \leq \ell\left(Q^{\prime}\right) \leq \mathcal{D}_{(p-1,1)}(B)$ that internally avoids $X_{B}$. Similar as before $d_{G \backslash X}(x, y) \leq 1+\mathcal{D}(F)+\mathcal{D}_{(p-1,1)}(B)-1=$ $\mathcal{D}(F)+\mathcal{D}_{(p-1,1)}(B)$.

Example 4.3. Lemma 4.2 for $p=1$ reads:

$$
\mathcal{D}_{2}^{V}(G) \leq \mathcal{D}(F)+\max \left\{\mathcal{D}_{1}^{V}(B), \mathcal{D}_{1}^{E}(B)\right\}
$$


1. Let $B=K_{4} \backslash\{e\}$. Then $\mathcal{D}(B)=\mathcal{D}_{1}^{V}(B)=\mathcal{D}_{1}^{E}(B)=2$. The 2-vertex fault diameter of Cartesian graph product $P_{3} \square B$ is $\mathcal{D}_{2}^{V}\left(P_{3} \square B\right)=\mathcal{D}\left(P_{3}\right)+\mathcal{D}_{1}^{V}(B)=$ $2+2=4$.

2. The 2-vertex fault diameter of Cartesian graph product $P_{3} \square K_{3}$ is $\mathcal{D}_{2}^{V}\left(P_{3} \square K_{3}\right)=$ $\mathcal{D}\left(P_{3}\right)+\mathcal{D}_{1}^{E}\left(K_{3}\right)=2+2=4$.

In both examples the bound of Lemma 4.2 is tight.

Lemma 4.4. Let $G$ be a Cartesian graph bundle with fibre $F$ over the base graph $B$, the graph $F$ be a connected graph with diameter $\mathcal{D}(F)>1$, and the graph $B$ be $(0, q)+$ connected, $q>0$. Then

$$
\mathcal{D}_{(1, q)}(G) \leq \mathcal{D}(F)+\mathcal{D}_{q}^{E}(B)=\mathcal{D}(F)+\mathcal{D}_{(0, q)}(B)
$$

Proof. Let $F$ be a connected graph, $D(F)>1$, and $B$ be $(0, q)+$ connected graph, $q>0$. Then $\mathcal{D}_{q}^{E}(B) \geq 2$ and by Theorem 3.4, the Cartesian graph bundle $G$ with fibre $F$ over the base graph $B$ is $(1, q)+$ connected. Let $a \in V(G)$ be the faulty vertex and $Y \subseteq E(G)$ be the set of faulty edges, $|Y|=q$. Denote the set of degenerate edges in $Y$ by $Y_{D}$, and the set of nondegenerate edges by $Y_{N}, Y=Y_{N} \cup Y_{D}, p\left(Y_{D}\right) \subseteq V(B), p\left(Y_{N}\right) \subseteq E(B)$. Denote the set of faulty elements by $X=\{a\} \cup Y$. Let $x, y \in V(G) \backslash\{a\}$ be two arbitrary distinct nonfaulty vertices in $G$. We shall find an upper bound for the distance $d_{G \backslash X}(x, y)$.

- Suppose first that $x$ and $y$ are in the same fibre, i.e. $p(x)=p(y)$.

If $\left|F_{x} \cap X\right|=0$, then $d_{G \backslash X}(x, y) \leq \mathcal{D}(F)$. If $\left|F_{x} \cap X\right|>0$, then outside of fibre $F_{x}$ there are at most $q$ faulty elements. As the graph $B$ is $(0, q)+$ connected, there are at least $q+1$ neighbors of vertex $p(x)$ in $B$. Therefore there exist a neighbor $v$ of vertex $p(x)$ in $B$, such that $p(x) v \notin p\left(Y_{N}\right)$ and $\left|F(v) \cap\left(\{a\} \cup Y_{D}\right)\right|=0$, and there is a path $x \rightarrow x^{\prime} \stackrel{P}{\rightarrow} y^{\prime} \rightarrow y$ which avoids $X$, where $x^{\prime}, y^{\prime} \in F(v)$ and $\ell(P) \leq \mathcal{D}(F)$. Thus $d_{G \backslash X}(x, y) \leq 1+\mathcal{D}(F)+1 \leq \mathcal{D}(F)+\mathcal{D}_{q}^{E}(B)$.

- Now assume that $x$ and $y$ are in distinct fibres, i.e. $p(x) \neq p(y)$.

Let $B^{\prime}=B \backslash p\left(Y_{N}\right)$. As $\left|p\left(Y_{N}\right)\right| \leq q-\left|Y_{D}\right|$, the subgraph $B^{\prime}$ is at least $\left(0,\left|Y_{D}\right|\right)+$ connected and $p^{-1}\left(B^{\prime}\right)$ does not contain nondegenerate faulty edges, $\left|p^{-1}\left(B^{\prime}\right) \cap Y_{N}\right|=0$.

Let $Y^{\prime}=\left\{p(x) v \in E\left(B^{\prime}\right) ;\left|F(v) \cap\left(\{a\} \cup Y_{D}\right)\right|>0\right\}$. We distinguish two cases.

1. Let $a \in V\left(F_{x}\right) \cup V\left(F_{y}\right)$, and without of loss of generality assume $a \in V\left(F_{x}\right)$. Then $\left|Y^{\prime}\right| \leq\left|Y_{D}\right|$ and the subgraph $B^{\prime} \backslash Y^{\prime}=B \backslash\left(Y^{\prime} \cup p\left(Y_{N}\right)\right)$ is a connected graph. Therefore there exists a path $Q$ from $p(x)$ to $p(y)$ in $B$ of length $1 \leq$ $\ell(Q) \leq \mathcal{D}_{q}^{E}(B)$, that avoids $p\left(Y_{N}\right)$, and for the neighbor $v \in V(Q)$ of the vertex $p(x), e=p(x) v$, there is no faulty elements in the fibre $F(v)$. Note that the path $Q \backslash e$ avoids $p(a)$.

If $v=p(y)$ there is a path $x \rightarrow x^{\prime} \stackrel{P}{\rightarrow} y$, where $x^{\prime} \in F_{y}$ and $\ell(P) \leq \mathcal{D}(F)$, that avoids faulty elements, thus $d_{G \backslash X}(x, y) \leq 1+\mathcal{D}(F)<\mathcal{D}(F)+\mathcal{D}_{q}^{E}(B)$.

If $v \neq p(y)$ there is a path $x \rightarrow x^{\prime} \stackrel{P}{\rightarrow} y^{\prime} \stackrel{\tilde{Q} \backslash e}{\rightarrow} y$, where $x^{\prime}, y^{\prime} \in F(v)$ and $\ell(P) \leq \mathcal{D}(F)$, which avoids faulty elements, thus $d_{G \backslash X}(x, y) \leq 1+\mathcal{D}(F)+$ $\mathcal{D}_{q}^{E}(B)-1=\mathcal{D}(F)+\mathcal{D}_{q}^{E}(B)$.

2. If $a \notin V\left(F_{x}\right) \cup V\left(F_{y}\right)$, we distinguish three cases. 
(a) Suppose $\left|\left(F_{x} \cup F_{y}\right) \cap Y_{D}\right|=0$. There exist a path $Q$ from $p(x)$ to $p(y)$ in $B^{\prime} \subseteq B$ of length $\ell(Q) \leq \mathcal{D}_{q}^{E}(B)$, that avoids $p\left(Y_{N}\right)$. By Lemma 4.1 there are two internally vertex-disjoint paths from $x$ to $y$ in $p^{-1}(Q)$, that avoid $Y$ and at least one of them avoids faulty vertex $a$, thus $d_{G \backslash X}(x, y) \leq$ $\ell(Q)+D(F) \leq \mathcal{D}_{q}^{E}(B)+D(F)$.

(b) Suppose, that exactly one of fibres $F_{x}, F_{y}$ contains faulty edges, without of loss of generality let $\left|F_{x} \cap Y_{D}\right|>0$ and $\left|F_{y} \cap Y_{D}\right|=0$. Then $\left|Y^{\prime}\right| \leq\left|Y_{D}\right|$ and the subgraph $B^{\prime} \backslash Y^{\prime}=B \backslash\left(Y^{\prime} \cup p\left(Y_{N}\right)\right)$ is a connected graph. There exist a path $Q$ from $p(x)$ to $p(y)$ in $B$ of length $1 \leq \ell(Q) \leq \mathcal{D}_{q}^{E}(B)$, that avoids $p\left(Y_{N}\right)$ and for the neighbor $v \in Q$ of vertex $p(x), e=p(x) v$, there is no faulty elements in the fibre $F(v)$.

If $v=p(y)$ then $d_{G \backslash X}(x, y) \leq 1+\mathcal{D}(F)<\mathcal{D}(F)+\mathcal{D}_{q}^{E}(B)$.

If $v \neq p(y)$, let $v^{\prime} \in F(v)$ be a neighbor of $x$. As $\left|\left(F(v) \cup F_{y}\right) \cap Y_{D}\right|=0$, similar as in (a) there is a path from $v^{\prime}$ to $y$ in $p^{-1}(Q \backslash e)$ of length at most $\mathcal{D}(F)+\mathcal{D}_{q}^{E}(B)-1$, that avoids faulty elements, thus $d_{G \backslash X}(x, y) \leq$ $1+\mathcal{D}(F)+\mathcal{D}_{q}^{E}(B)-1=\mathcal{D}(F)+\mathcal{D}_{q}^{E}(B)$.

(c) At last, suppose $\left|F_{x} \cap Y_{D}\right|>0$ and $\left|F_{y} \cap Y_{D}\right|>0$.

i. Assume $d_{B^{\prime}}(p(x), p(y))=1$. In this case $p(x) p(y) \in Y^{\prime}$, and $\left|Y^{\prime}\right| \leq$ $\left|Y_{D}\right|$ as $\left|F_{x} \cap Y_{D}\right|>0$. Thus the subgraph $B^{\prime} \backslash Y^{\prime}$ is connected, and there exists a path $Q$ from $p(x)$ to $p(y)$ in $B$ of length $2 \leq \ell(Q) \leq$ $\mathcal{D}_{q}^{E}(B)$, that avoids $p\left(Y_{N}\right)$, and for the neighbor $v \in Q$ of vertex $p(x)$, $e=p(x) v$, there is no faulty elements in the fibre $F(v)$.

If $\ell(Q)=2$, then there is a path $x \rightarrow x^{\prime} \stackrel{P}{\rightarrow} y^{\prime} \rightarrow y$, where $x^{\prime}, y^{\prime} \in$ $F(v)$ and $\ell(P) \leq \mathcal{D}(F)$, which avoids faulty elements, thus $d_{G \backslash X}(x, y) \leq 1+\mathcal{D}(F)+1 \leq \mathcal{D}(F)+\mathcal{D}_{q}^{E}(B)$.

If $3 \leq \ell(Q) \leq \mathcal{D}_{q}^{E}(B)$, then the path $x \rightarrow x^{\prime} \stackrel{P^{\prime}}{\rightarrow} y^{\prime} \rightarrow s \rightarrow y$ where $x^{\prime}, y^{\prime} \in F(v), \ell\left(P^{\prime}\right) \leq \mathcal{D}(F)$, and $s \in V\left(F_{x}\right)$, avoids faulty elements, thus $d_{G \backslash X}(x, y) \leq 1+\mathcal{D}(F)+1+1 \leq \mathcal{D}(F)+\mathcal{D}_{q}^{E}(B)$.

ii. Assume, $d_{B^{\prime}}(p(x), p(y))=2$. Then there is at least one common neighbor of vertices $p(x)$ and $p(y)$ in $B^{\prime}$. If there exist a common neighbor $v$ of vertices $p(x)$ and $p(y)$ in $B^{\prime}$ for which there is no faulty elements in the fibre $F(v)$, then as before $d_{G \backslash X}(x, y) \leq 1+\mathcal{D}(F)+$ $1 \leq \mathcal{D}(F)+\mathcal{D}_{q}^{E}(B)$. Otherwise suppose, there is some common neighbor $w$ of vertices $p(x)$ and $p(y)$ in $B^{\prime}$ for which $a \notin F(w)$ and $\left|F(w) \cap Y_{D}\right|>0$. As $\left|Y^{\prime}\right| \leq\left|Y_{D}\right|-1$ the subgraph $B^{\prime} \backslash Y^{\prime}$ is (at least) $(0,1)+$ connected graph. If vertex $p(a)$ is a neighbor of $p(y)$ in $B^{\prime}, e^{\prime}=p(y) p(a) \subseteq E\left(B^{\prime}\right)$, then also $B^{\prime} \backslash\left(Y^{\prime} \cup\left\{e^{\prime}\right\}\right)$ is a connected graph. Therefore there exist a path $Q$ from $p(x)$ to $p(y)$ in $B$ of length $3 \leq \ell(Q) \leq \mathcal{D}_{q}^{E}(B)$, that avoids $p\left(Y_{N}\right)$, and for the neighbor $u \in$ $V(Q)$ of vertex $p(x)$, there is no faulty elements in the fibre $F(u)$, and for the neighbor $v \in V(Q)$ of vertex $p(y), v \neq p(a)$.

If $\ell(Q)=3 \leq \mathcal{D}_{q}^{E}(B)$, then there is a path $x \rightarrow x^{\prime} \stackrel{P}{\rightarrow} y^{\prime} \rightarrow s \rightarrow y$, where $x^{\prime}, y^{\prime} \in F(u), \ell(P) \leq \mathcal{D}(F), s \in F(v)$, which avoids faulty elements, thus $d_{G \backslash X}(x, y) \leq 1+\mathcal{D}(F)+1+1 \leq \mathcal{D}(F)+\mathcal{D}_{q}^{E}(B)$. If $4 \leq \ell(Q) \leq \mathcal{D}_{q}^{E}(B)$, then the path $x \rightarrow x^{\prime} \stackrel{P^{\prime}}{\rightarrow} y^{\prime} \rightarrow s \rightarrow s^{\prime} \rightarrow y$, where $x^{\prime}, y^{\prime} \in F(u), \ell\left(P^{\prime}\right) \leq \mathcal{D}(F), s \in V\left(F_{x}\right), s^{\prime} \in F(w)$, avoids 
faulty elements, thus $d_{G \backslash X}(x, y) \leq 1+\mathcal{D}(F)+1+2 \leq \mathcal{D}(F)+$ $\mathcal{D}_{q}^{E}(B)$.

The last case to consider is when $p(a)$ is the only common neighbor of vertices $p(x)$ and $p(y)$ in $B^{\prime}$.

Let $Y^{\prime \prime}=\left\{p(y) v \in E\left(B^{\prime}\right) ;\left|F(v) \cap\left(\{a\} \cup Y_{D}\right)\right|>0\right\}$. As $p(a)$ is the only common neighbor of vertices $p(x)$ and $p(y),\left|Y^{\prime} \cup Y^{\prime \prime}\right| \leq$ $\left|Y_{D}\right|$ and the subgraph $B^{\prime} \backslash\left(Y^{\prime} \cup Y^{\prime \prime}\right)$ is a connected graph. Therefore there exist a path $Q$ from $p(x)$ to $p(y)$ in $B$ of length $3 \leq \ell(Q) \leq$ $\mathcal{D}_{q}^{E}(B)$, that avoids $p\left(Y_{N}\right)$, and for neighbors $u \in V(Q)$ of vertex $p(x)$ and $v \in V(Q)$ of vertex $p(y)$, there is no faulty elements in fibres $F(u)$ and $F(v)$. Let $x^{\prime} \in F(u)$ be a neighbor of $x$ and $y^{\prime} \in$ $F(v)$ be a neighbor of $y$. As in (a) there is a path from $x^{\prime}$ to $y^{\prime}$ in $p^{-1}\left(Q \backslash\{p(x), p(y)\}\right.$ of length at most $\mathcal{D}(F)+\mathcal{D}_{q}^{E}(B)-2$, that avoids faulty elements, thus $d_{G \backslash X}(x, y) \leq 1+\mathcal{D}(F)+\mathcal{D}_{q}^{E}(B)-2+1=$ $\mathcal{D}(F)+\mathcal{D}_{q}^{E}(B)$.

iii. Finally, suppose $d_{B^{\prime}}(p(x), p(y)) \geq 3$. As there is no common neighbor of vertices $p(x)$ and $p(y)$ in $B^{\prime},\left|Y^{\prime} \cup Y^{\prime \prime}\right| \leq\left|Y_{D}\right|-2+1=$ $\left|Y_{D}\right|-1$ and as before there exist a path $Q$ from $p(x)$ to $p(y)$ in $B$ of length $3 \leq \ell(Q) \leq \mathcal{D}_{q}^{E}(B)$, that avoids $p\left(Y_{N}\right)$, and for both neighbors $u \in V(Q)$ of vertex $p(x)$ and $v \in V(Q)$ of vertex $p(y)$, there is no faulty elements in fibres $F(u) \cup F(v)$, thus $d_{G \backslash X}(x, y) \leq$ $1+\mathcal{D}(F)+\mathcal{D}_{q}^{E}(B)-2+1=\mathcal{D}(F)+\mathcal{D}_{q}^{E}(B)$.

Lemma 4.5. Let $G$ be a Cartesian graph bundle with fibre $F$ over the base graph $B$, the graph $F$ be a connected graph with diameter $\mathcal{D}(F)>1$, and the graph $B$ be $(p, q)+$ connected, $q>0$. Then

$$
\mathcal{D}_{(p+1, q)}(G) \leq \mathcal{D}(F)+\mathcal{D}_{(p, q)}(B)
$$

Proof. The case when $p=0$ is already proved by Lemma 4.4. So let us assume $p>0$. Let the graph $F$ be a connected graph, $\mathcal{D}(F)>1$, and the graph $B$ be $(p, q)+$ connected, $p, q>0$. Then $\mathcal{D}_{(p, q)}(B) \geq 2$ and by Theorem 3.4, the Cartesian graph bundle $G$ with fibre $F$ over the base graph $B$ is $(p+1, q)+$ connected. Let $X \subseteq V(G)$ be the set of faulty vertices, $|X|=p+1$, and $Y \subseteq E(G)$ be the set of faulty edges, $|Y|=q$. Denote the set of degenerate edges in $Y$ by $Y_{D}$, and the set of nondegenerate edges by $Y_{N}, Y=Y_{N} \cup Y_{D}$, $p\left(Y_{D}\right) \subseteq V(B), p\left(Y_{N}\right) \subseteq E(B)$. Let $x, y \in V(G) \backslash X$ be two distinct nonfaulty vertices in $G$. We shall determine an upper bound for the distance $d_{G \backslash(X \cup Y)}(x, y)$.

- Suppose first that $x$ and $y$ are in the same fibre, i.e. $p(x)=p(y)$.

If $\left|F_{x} \cap\left(X \cup Y_{D}\right)\right|=0$, then $d_{G \backslash(X \cup Y)}(x, y) \leq \mathcal{D}(F)$. If $\left|F_{x} \cap\left(X \cup Y_{D}\right)\right|>0$, then there are at most $p+q$ faulty elements outside of the fibre $F_{x}$. As the graph $B$ is $(p, q)+$ connected, there are at least $p+q+1$ neighbors of vertex $p(x)$ in $B$. Therefore there exists a neighbor $v$ of vertex $p(x)$ in $B$, such that $p(x) v \notin p\left(Y_{N}\right)$ and $\left|F(v) \cap\left(X \cup Y_{D}\right)\right|=0$, and there is a path $x \rightarrow x^{\prime} \stackrel{P}{\rightarrow} y^{\prime} \rightarrow y$, where $x^{\prime}, y^{\prime} \in F(v)$ and $\ell(P) \leq \mathcal{D}(F)$, which avoids $X \cup Y$. Thus $d_{G \backslash(X \cup Y)}(x, y) \leq 1+\mathcal{D}(F)+1 \leq$ $\mathcal{D}(F)+\mathcal{D}_{(p, q)}(B)$. 
- Now assume that $x$ and $y$ are in distinct fibres, i.e. $p(x) \neq p(y)$.

Let $X_{B}=\{v \in V(B) \backslash\{p(x), p(y)\} ;|F(v) \cap X|>0\}$. We distinguish two cases.

1. If $p \leq\left|X_{B}\right| \leq p+1$, then let $X_{B}^{\prime} \subseteq X_{B},\left|X_{B}^{\prime}\right|=p$. The subgraph $B \backslash X_{B}^{\prime}$ is $(0, q)+$ connected and there is at most one faulty vertex and $q$ faulty edges in $p^{-1}\left(B \backslash X_{B}^{\prime}\right)$. By Lemma 4.4 there is a path from $x$ to $y$ in $p^{-1}\left(B \backslash X_{B}^{\prime}\right)$ with length at most $\mathcal{D}(F)+\mathcal{D}_{q}^{E}\left(B \backslash X_{B}^{\prime}\right)$, that avoids faulty elements, thus $d_{G \backslash(X \cup Y)}(x, y) \leq \mathcal{D}(F)+\mathcal{D}_{q}^{E}\left(B \backslash X_{B}^{\prime}\right)=\mathcal{D}(F)+\mathcal{D}_{(p, q)}(B)$.

2. Suppose $\left|X_{B}\right|<p$. Let $Y_{B}=\left\{p(x) v \in E(B) ;\left|F(v) \cap Y_{D}\right|>0\right\}$ and $B^{\prime}=$ $B \backslash\left(X_{B} \cup Y_{B} \cup p\left(Y_{N}\right)\right)$. Then the subgraph $B^{\prime}$ is (at least) $(1,0)+$ connected, thus also $(0,1)+$ connected.

If $d_{B^{\prime}}(p(x), p(y)) \geq 2$, then there is a path $Q$ from $p(x)$ to $p(y)$ in $B^{\prime} \subseteq B$ with $2 \leq \ell(Q) \leq \mathcal{D}_{(p-1, q)}(B) \leq \mathcal{D}_{(p, q)}(B)$ that internally avoids $X_{B}$, it avoids $p\left(Y_{N}\right)$, and for the neighbor $v \in V(Q)$ of vertex $p(x), e^{\prime}=p(x) v$, there is no faulty elements in the fibre $F(v)$. Therefore there is a path $x \rightarrow$ $x^{\prime} \stackrel{P}{\rightarrow} y^{\prime} \stackrel{\tilde{Q} \backslash e^{\prime}}{\rightarrow} y$, where $x^{\prime}, y^{\prime} \in F(v)$ and $\ell(P) \leq \mathcal{D}(F)$, which avoids $X \cup Y$, thus $d_{G \backslash X \cup Y}(x, y) \leq 1+\mathcal{D}(F)+\mathcal{D}_{(p, q)}(B)-1=\mathcal{D}(F)+\mathcal{D}_{(p, q)}(B)$.

If $d_{B^{\prime}}(p(x), p(y))=1, e=p(x) p(y) \in E\left(B^{\prime}\right)$, then the subgraph $B^{\prime} \backslash\{e\}$ is a connected graph and there is a path $Q^{\prime}$ from $p(x)$ to $p(y)$ with $2 \leq \ell\left(Q^{\prime}\right) \leq$ $\mathcal{D}_{(p-1, q+1)}(B) \leq \mathcal{D}_{(p, q)}(B)$ that internally avoids $X_{B}$, it avoids $p\left(Y_{N}\right)$, and for the neighbor $v \in V(Q)$ of vertex $p(x)$, there is no faulty elements in the fibre $F(v)$, and as before $d_{G \backslash X \cup Y}(x, y) \leq 1+\mathcal{D}(F)+\mathcal{D}_{(p, q)}(B)-1=$ $\mathcal{D}(F)+\mathcal{D}_{(p, q)}(B)$.

Theorem 4.6. Let $G$ be a Cartesian graph bundle with fibre $F$ over the base graph $B$, the graph $F$ be a connected graph with diameter $\mathcal{D}(F)>1$, and the graph $B$ be $(p, q)+$ connected, $p+q>0$. Then we have:

- If $q>0$, then $\mathcal{D}_{(p+1, q)}(G) \leq \mathcal{D}(F)+\mathcal{D}_{(p, q)}(B)$.

- If $q=0$, then $\mathcal{D}_{(p+1,0)}(G)=\mathcal{D}_{p+1}^{V}(G) \leq \mathcal{D}(F)+\max \left\{\mathcal{D}_{p}^{V}(B), \mathcal{D}_{(p-1,1)}(B)\right\}$.

Proof. The statement of Theorem 4.6 follows from Lemma 4.2 (case $q=0$ ), Lemma 4.4 (case $p=0$ ), and Lemma 4.5 (for positive $p$ and $q$ ).

Remark 4.7. Let $G$ be a Cartesian graph bundle with fibre $F$ over the base graph $B$, the graph $F$ be a connected graph with diameter $\mathcal{D}(F)>1$, and the graph $B$ be $(p, 0)+$ connected, $p>0$. By Theorem 4.6 we have an upper bound for the (vertex) fault diameter $\mathcal{D}_{p+1}^{V}(G) \leq \mathcal{D}(F)+\mathcal{D}_{p}^{V}(B)+1$ for any graph $B$. Similarly, $\mathcal{D}_{p+1}^{V}(G) \leq \mathcal{D}(F)+\mathcal{D}_{p}^{V}(B)$ if $\mathcal{D}_{(p-1,1)}(B) \leq \mathcal{D}_{p}^{V}(B)$ holds.

Next corollary easily follows from Theorems 3.5 and 4.6.

Corollary 4.8. Let both graphs $F$ and $B$ be $(p, q)+$ connected, $p+q>0, \mathcal{D}(F)>1$, $\mathcal{D}(B)>1$, and let $G$ be a Cartesian graph bundle with fibre $F$ over the base graph $B$. Then we have:

- If $q>0$, then $\mathcal{D}_{(p+1, q)}(G) \leq \max \left\{\mathcal{D}(F)+\mathcal{D}_{(p, q)}(B), \mathcal{D}_{(p, q)}(F)+\mathcal{D}(B)\right\}$, 
- If $q=0$, then $\mathcal{D}_{p+1}^{V}(G) \leq \max \left\{\mathcal{D}(F)+\mathcal{D}_{p}^{V}(B), \mathcal{D}_{p}^{V}(F)+\mathcal{D}(B)\right\}+1$, and $\mathcal{D}_{p+1}^{V}(G) \leq \max \left\{\mathcal{D}(F)+\mathcal{D}_{p}^{V}(B), \mathcal{D}_{p}^{V}(F)+\mathcal{D}(B)\right\}$, if $\mathcal{D}_{(p-1,1)}(F) \leq \mathcal{D}_{p}^{V}(F)$ and $\mathcal{D}_{(p-1,1)}(B) \leq \mathcal{D}_{p}^{V}(B)$ hold.

We conclude with a conjecture. We know that a Cartesian graph bundle with fibre $F$ over the base graph $B$, where graph $F$ is $\left(p_{F}, q_{F}\right)+$ connected, $p_{F}+q_{F}>0$, and where graph $B$ is $\left(p_{B}, q_{B}\right)+$ connected, $p_{B}+q_{B}>0$, is $\left(p_{B}+p_{F}+1, q_{B}+q_{F}\right)+$ connected [13]. An upper bound for the mixed fault diameter where the number of allowed faulty elements would be the maximal possible may be the following:

Conjecture 4.9. Let $G$ be a Cartesian graph bundle with fibre $F$ over the base graph $B$, where the graph $F$ is $\left(p_{F}, q_{F}\right)+$ connected, $p_{F}+q_{F}>0$, and where the graph $B$ is $\left(p_{B}, q_{B}\right)+$ connected, $p_{B}+q_{B}>0$. Then

$$
\mathcal{D}_{\left(p_{B}+p_{F}+1, q_{B}+q_{F}\right)}(G) \leq \mathcal{D}_{\left(p_{F}, q_{F}\right)}(F)+\mathcal{D}_{\left(p_{B}, q_{B}\right)}(B)+1 .
$$

\section{References}

[1] J. M. Aldous, R. J. Wilson, Graphs and Applications: An introductory Approach, Springer, Berlin, 2000.

[2] I. Banič, J. Žerovnik, Fault-diameter of Cartesian graph bundles, Inform. Process. Lett. 100 (2006), 47-51.

[3] I. Banič, J. Žerovnik, Edge fault-diameter of Cartesian product of graphs, Lect. Notes Comput. Sc. 4474 (2007), 234-245.

[4] I. Banič, J. Žerovnik, Fault-diameter of Cartesian product of graphs, Adv. Appl. Math. 40 (2008), 98-106.

[5] I. Banič, R. Erveš, J. Žerovnik, The edge fault-diameter of Cartesian graph bundles, Eur. J. Combin. 30 (2009), 1054-1061.

[6] I. Banič, R. Erveš, J. Žerovnik, Edge, vertex and mixed fault diameters, Adv. Appl. Math. 43 (2009), 231-238.

[7] L. W. Beineke, F. Harary, The connectivity function of a graph, Mathematika 14 (1967), 197202.

[8] J.-C. Bermond, N. Honobono, C. Peyrat, Large Fault-tolerant Interconnection Networks, Graph. Combinator. 5 (1989), 107-123.

[9] P. Cull, S. M. Larson, On generalized twisted cubes, Inform. Process. Lett. 55 (1995), 53-55.

[10] K. Day, A. Al-Ayyoub, Minimal fault diameter for highly resilient product networks, IEEE T. Parallel. Distr. 11 (2000), 926-930.

[11] D. Z. Du, D. F. Hsu, Y. D. Lyuu, On the diameter vulnerability of kautz digraphs, Discrete Math. 151 (2000), 81-85.

[12] K. Efe, A variation on the hypercube with lower diameter, IEEE T. Comput. 40 (1991), 1312 1316.

[13] R. Erveš, J. Žerovnik, Mixed connectivity of Cartesian graph products and bundles, to appear in Ars Combinatoria, arXiv:1002.2508v1 [math.CO] (2010).

[14] R. Erveš, J. Žerovnik, Mixed fault diameter of Cartesian graph bundles, Discrete Appl. Math. 161 (2013), 1726-1733, doi:10.1016/j.dam.2011.11.020.

[15] R. Hammack, W. Imrich, S. Klavžar, Handbook of Product Graphs, second ed., CRC Press, 2011. 
[16] C. H. Hung, L. H. Hsu, T. Y. Sung, On the Construction of Combined $k$-Fault-Tolerant Hamiltonian Graphs, Networks 37 (2001), 165-170.

[17] W. Imrich, T. Pisanski, J. Žerovnik, Recognizing Cartesian graph bundles, Discrete Math. 167168 (1997), 393-403.

[18] M. Krishnamoorthy, B. Krishnamurty, Fault diameter of interconnection networks, Comput. Math. Appl. 13 (1987), 577-582.

[19] S. C. Liaw, G. J. Chang, F. Cao, D. F. Hsu, Fault-tolerant routing in circulant networks and cycle prefix networks, Ann. Comb. 2 (1998), 165-172.

[20] W. Mader, Connectivity and edge-connectivity infinite graphs, Surveys in Combinatorics. Lond. Math. S. 38 (1979), 66-95.

[21] T. Pisanski, J. Vrabec, Graph bundles, Preprint Series Department of Mathematics, vol. 20, no. 079, p. 213-298, Ljubljana, 1982.

[22] T. Pisanski, J. Shawe-Taylor, J. Vrabec, Edge-colorability of graph bundles, J. Comb. Theory B 35 (1983), 12-19.

[23] I. Stojmenović, Multiplicative circulant networks: Topological properties and communication algorithms, Discrete Appl. Math. 77 (1997), 281-305.

[24] C. M. Sun, C. N. Hung, H. M. Huang, L. H. Hsu, Y. D. Jou, Hamiltonian Laceability of Faulty Hypercubes, Journal of Interconnection Networks 8 (2007), 133-145.

[25] M. Xu, J.-M. Xu, X.-M. Hou, Fault diameter of Cartesian product graphs, Inform. Process. Lett. 93 (2005), 245-248.

[26] J. H. Yin, J. S. Li, G. L. Chen, C. Zhong, On the Fault-Tolerant Diameter and Wide diameter of $\omega$-Connected Graphs, Networks 45 (2005), 88-94.

[27] B. Zmazek, J. Žerovnik, Recognizing weighted directed Cartesian graph bundles, Discussiones Mathematicae Graph Theory 20 (2000), 39-56.

[28] B. Zmazek, J. Žerovnik, On recognizing Cartesian graph bundles, Discrete Math. 233 (2001), 381-391.

[29] B. Zmazek, J. Žerovnik, Algorithm for recognizing Cartesian graph bundles, Discrete Appl. Math. 120 (2002), 275-302.

[30] B. Zmazek, J. Žerovnik, Unique square property and fundamental factorizations of graph bundles, Discrete Math. 244 (2002), 551-561.

[31] J. Žerovnik, On recognizing of strong graph bundles, Math. Slovaca 50 (2000), 289-301. 